\title{
Error Correction of Temperature Measurement System Based on the Seepage Test
}

\author{
Siling Liang, Jiawei Mi, Yutong Cao \\ Hohai University, NanJing, China \\ liangsiling@yahoo.com
}

Keyword: Error, Temperature, Correction model.

\begin{abstract}
This study is based on the application of temperature in seepage experiment. The output temperature is compared with the actual temperature to fit the curve of the relationship through the measured data of the 107 groups. The error correction model is established to achieve accurate measurement of sensor. After verification by experiment, the measured error is greatly reduced. The modified model can effectively weaken the influence of temperature on the measurement precision.
\end{abstract}

\section{Introduction}

Leakage and piping are the main form of embankment danger in various factors. Temperature tracer can use the temperature parameters of leakage water to obtain the size parameters and leakage flow rate. Therefore, the accurate measurement of temperature has important significance in the research of seepage test. The temperature parameter obtained by the temperature sensor used in the test is analog. Besides there are differences between each sensor, the length of wires attached to it, and the output error in digital temperature recorder. Therefore, the error correction for the temperature measurement system is quite significant.

\section{Design of temperature fitting test}

The test process. Test equipment: industrial mercury thermometer at 0 to 50 DEG $\mathrm{C}$ range; the temperature sensor of LM35 (exists a built-in integrated circuit and a wider range of linear working range; due to the use of internal compensation, the output starts from 0 DEG C) [1,2,3]; the number controlled thermostatic bath of SC-15(the temperature adjustment range is from room temperature to $100 \mathrm{DEG} \mathrm{C}$, the display resolution is 0.1 DEG C); the digital temperature recorder of SY-5(the reading accuracy is 0.1 DEG C; the range is from -30 DEG C to +150 DEG C).

Prior to the start of the trial, reform 107 groups of sensors separately for waterproof. The sensor is pasted with epoxy resin and covered by a heat shrink tubing. For the convenience of the experimental operation, three wires connected to the sensor respectively should stick on rubberized fabric of three different colors for visual differentiation. Among those the groups of sensors from No.1 to No.61 connect with one-mile-long wires while the groups of sensors from No.62 to No.107 connect with two-mile-long wires. The rest of the characteristics of the 107 groups of sensors are the same.

Test procedure: (a) Number the sensors in which the number $1-\mathrm{N}(1<=\mathrm{N}<=61)$ indicates the Nth sensor connected with one-mile-long wires and the number $2-\mathrm{N}(1<=\mathrm{N}<=46)$ indicates the Nth sensor connected with two-mile-long wires. (b)Turn the thermostatic bath on 0 DEG $\mathrm{C}$ to measure the data. Then set 5 DEG C as a gradient to raise the temperature until 50 DEG C. Record 107 groups of reading every time after change the temperature of the thermostatic bath. The measurement time should be controlled within 5 minutes. 
The analysis of experimental data. During the measurement, the actual temperature measured by the thermometer is denoted by $T_{1}$, and the experimental temperature measured by digital temperature recorder is denoted by $T_{2} . T_{1}$ and $T_{2}$ changes at the same time when the temperature changes suddenly. All groups of the fitting data of the actual temperature and the experimental temperature should be carried on separately. The relationship of them can be described by a linear function:

$$
\mathrm{T}_{2}=\mathrm{n}^{*} \quad \mathrm{~T}_{1}+\mathrm{a}
$$

Collect the slope to conclude that the average slope is $\mathrm{n} 1=0.993$ with the wires of length $\mathrm{L}=1 \mathrm{~m}$ while the average slope is $n 1=1.056$ with the wires of the length $\mathrm{L}=2 \mathrm{~m}$. Error increment of the group of $\mathrm{L}=1 \mathrm{~m}$ is less than the group of $\mathrm{L}=2 \mathrm{~m}$ when the experimental temperature is compared with the actual temperature.

$\mathrm{T}_{1}$ and $\mathrm{T}_{2}$ of 107 groups are fitted for the quadratic function curve respectively. Then differentiate quadratic function to obtain the slope change rate for each curve. Collect 107 groups of the data of the change rate in order to make the cumulative distribution curve of slope change rate of two different types of groups as in Figure 1 and Figure 2. At the same time, the average of change rate are $n_{1}{ }^{\prime}=0.00194$ and $n_{2}{ }^{\prime}=0.00365$ by calculation, which indicates that the rate of error growth of $\mathrm{L}=2 \mathrm{~m}$-groups is roughly twice of $\mathrm{L}=1 \mathrm{~m}$-groups in velocity in the process the temperature increases from 0 DEG C to 50 DEG C.

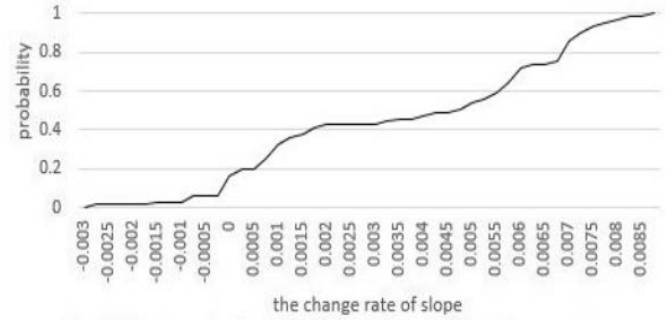

Figure 1 The cumulative distributing curve of change rate of slope $(\mathrm{L}=1 \mathrm{~m})$

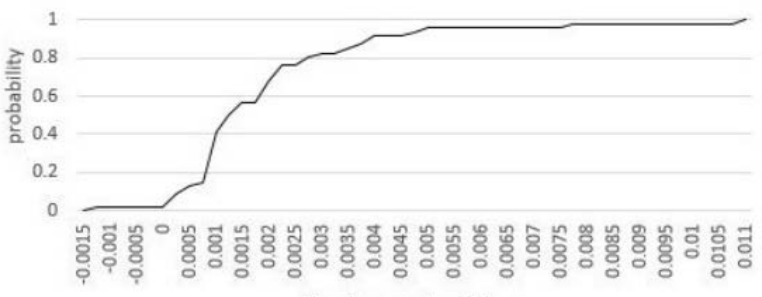

Figure 2 The cumulative distributing curve of change rate of slope $(\mathrm{L}=2 \mathrm{~m})$

The slope change rate in Figure 1 is evenly distributed in the range from -0.003 to 0.0085 . And the error growth rate of $\mathrm{L}=1 \mathrm{~m}$-groups can be regarded as a whole growth trend for the probability in the range from -0.003 to 0 is approximately equal to 0 . Also, the curve in Figure 2 has a similar growth trend. According to above analysis, raising the temperature can accelerate the increase of error.

\section{The correction model of the temperature}

Fit formula of $\mathrm{T} 1$ and $\mathrm{T} 2$ as follows:

$$
\mathrm{T}_{2}=\mathrm{m} * \mathrm{~T}_{1}+\mathrm{b}
$$

Integrate all the 107 groups of fitting results to achieve the correction model of T1 and T2. When $\mathrm{L}=1 \mathrm{~m}$ that $\mathrm{T}_{0}=0.9991 * \mathrm{~T}_{2}-0.6197$ while $\mathrm{L}=2 \mathrm{~m}$ the $\mathrm{T}_{0}=0.9534 * \mathrm{~T}_{2}-1.096$. In which $\mathrm{T}_{0}$ is the theory temperature after error correction.

\section{The verification test}

Write the error correction model into the intelligent control chip of the temperature measurement system. Then select another temperature sensor with a one-mile-long wire and a temperature sensor with a two-mile-long wire. Put the two temperature sensors in the thermostatic bath and record the 
measured temperature at nine different temperatures in the range of $0^{\circ} \mathrm{C} \sim 50^{\circ} \mathrm{C}$. The output result of digital temperature recorder is denoted as the experimental temperature. And the reading of the thermometer is denoted as the actual temperature and the correction result of the temperature measurement system is denoted as the theory temperature.

Compare the fitting curve at nine different measured temperature as shown in Figure 8 and Figure 9. So the error between the experimental temperature and the actual temperature is large while the output data from the temperature measurement system and the actual temperature are basically in coincidence. This shows that the error correction model is quite effective which reduced the influence of temperature rising on measurement precision.
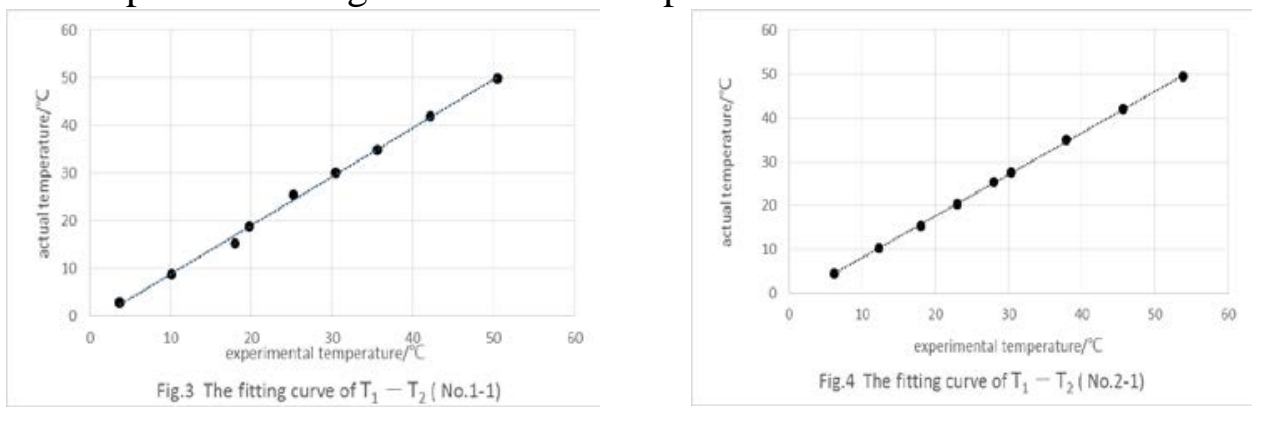

\section{Summary}

This paper analyzes the trend of error and study on the error correction model. Based on a large number of experimental data, the theory temperature and the actual temperature have a high fitting degree after correction m. Thus, the correction temperature can improve the accuracy of the system markedly. In actual application, it is necessary to measure the test data as much as possible to collect more data samples to improve the accuracy of the error correction model.

\section{References}

[1] SU Penghui, XU Yaguang, LI Cheng, SUN Yanfeng. Analysis of Automatic Test System Error for Temperature Sensor [J]. Electro-optic Technology Application, 2009, 24(3) 44-46.

[2] LU Jianguo. Design of the Digital Thermometer Based on LM35\& W78E52B [J]. Journal of Anhui Agriculture Science, 2010, 38(24) 13542-13546.

[3] SHEN Rui, YU Haixun, WANG Yaowen. Design of Temperature Acquisition System Based on ARM7 and LM35 [J]. Modern Electronics Technique, 2012,35 (6) 173-175.

[4] LIAO Li. Errors and Effect Factors Analysis of Temperature Sensor's Dynamic Response Characteristics Calibration [J]. Aviation Metrology \& Measurement Technology, 1997, 18 (1)21-23. 